\title{
Nanowire Grid Polarizers Integrated into Flexible, Gas Permeable, Biocompatible Materials and Contact Lenses
}

\author{
Andrew E. Hollowell and L. Jay Guo*
}

The integration of optical components directly into contact lens materials permits the realization of advanced display technologies and integrated medical instruments. A process is developed for the fabrication of aluminium nanowire grid polarizers encapsulated in a flexible, gas permeable, and biocompatible material for integration onto rigid, gas-permeable contact lenses. Gas permeability values an order of magnitude higher than the minimum to prevent eye damage are measured while maintaining strong polarization performance. Further, the methods developed in this work can readily be applied to various optical and electronic technologies, allowing standard fabrication processes on rigid substrates and subsequent transfer to flexible, gas permeable, and biocompatible materials.

or various processing chemicals (as are plastics and other flexible films), and then encapsulate and transfer these devices into flexible, gas permeable biocompatible films. As an example of this technique, we have fabricated biocompatible flexible nanowire grid polarizers (NWGPs) embedded in polydimethylsiloxane (PDMS), with measured oxygen transmissibility of up to $5.58 \times 10^{-7} \mathrm{Dk} / \mathrm{t}(\mathrm{cm} \mathrm{mL}$ $\left.\mathrm{O}_{2} / \mathrm{s} \mathrm{mL} \mathrm{mmHg}\right)\left(2.79 \times 10^{-9} \mathrm{Dk}\left(\mathrm{cm}^{2} \mathrm{~mL}\right.\right.$ $\mathrm{O}_{2} / \mathrm{s} \mathrm{mL} \mathrm{mmHg))} \mathrm{while} \mathrm{still} \mathrm{possessing}$ strong polarization performance.

Polarizers are widely used in optical systems for microscopy, imaging, and display technologies. Particularly, wire grid

\section{Introduction}

The integration of electronics and optical components with biocompatible materials will increase the functionality of the human body and expand the market for display, sensing, and medical fields. Display technologies have followed a constant trend to generate thinner, lighter weight, and flexible systems. A future step towards advancing displays is to integrate systems directly onto human eyes. Current heads-up display (HUD) systems are bulky and infringe upon comfort, mobility, and field of view. Integrating portions of HUD systems directly with contact lenses generates the possibility of compact, portable, and discrete displays that do not limit user functionality. The same ideology of integrating optical components into contact lens can be extended to further advancing medical instruments and sensors directly connected to a human body. Micro and nanofabrication processes have previously been developed to place semiconductor devices on flexible plastics and curved surfaces for integration into contact lenses. ${ }^{[1,2]}$ However, these materials are not gas permeable, leading to potential eye damage if worn for extended periods of time. In this work, we have developed a process that allows optical and electronic devices to be fabricated on rigid substrates, that are not sensitive to temperature

A. E. Hollowell, Prof. L. J. Guo

Department of Electrical and Computer Science

University of Michigan

1301 Beal Avenue, Ann Arbor, MI, 48109, USA

E-mail: guo@umich.edu

A. E. Hollowell

Sandia National Laboratories

Organization 01725 Photonic Microsystems

1515 Eubank Blvd

Albuquerque, NM, 87123, USA

DOI: 10.1002/adom.201300024 polarizers (WGPs), which consist of subwavelength periodic metallic lines, are easily integrated into optical systems. The spectrum of polarization for WGPs corresponds to the periodic spacing between metal lines; thus, historic research was first applied towards infrared wavelengths. ${ }^{[3]}$ With the advancement of micro and nanoscale fabrication techniques, WGPs have extended beyond IR wavelengths and reached the visible spectrum with very high extinction ratios and relative ease of fabrication. Compared to other types of polarizers, WGPs can be extremely thin, flexible, have long term stability and wide field of view while maintaining high polarization efficiency. ${ }^{[3,4]}$ Fabricating NWGPs for visible light requires subwavelength features (pitch < $200 \mathrm{~nm}$ ), which are difficult to fabricate on a large scale with conventional lithographic techniques. Electronbeam lithography offers low throughput, and immersion interferometric lithography has limited field size and strict process controls. ${ }^{[5]}$ In this work nanoimprint lithography (NIL) is used as an efficient technology for nanoscale patterning with high throughput and low cost. In contrast to liftoff processes which have limitations on line width and aspect ratio, oblique angled evaporations that require multiple depositions ${ }^{[6-9]}$ and transfer printing which has strict material requirements, ${ }^{[10,11]}$ a single step RIE into aluminium $(\mathrm{Al})^{[5,12,13]}$ through a nanoimprint defined mask is employed here as an efficient method to form an Al wire grid. PDMS is used as a flexible, biocompatible, and gas permeable material to encapsulate the NWGPs and extremely selective gas etching is utilized to release the structure from the carrier substrate. We have been successful in directly casting PDMS as well as transferring PDMS films onto rigid gas permeable (RGP) lenses, however, to our knowledge no one has fully encapsulated wire grid polarizers into PDMS. The methods developed here demonstrate a suitable process for large scale manufacturing of visible wavelength NWGPs integrated directly onto RGP contact lenses. 


\section{Design}

The designed structure consists of an Al wire grid released from its carrier substrate and encapsulated in PDMS, as shown in Figure 1. The most important structural parameters that determine the performance of a WGP, namely, the period, width, and height of the $\mathrm{Al}$ wire grid is denoted as $\mathrm{P}, \mathrm{W}$, and $\mathrm{H}$ respectively. PDMS is chosen to fill the open spacing between metallic lines due to its gas permeable and biocompatible material property, which allows the system to remain gas permeable. Encapsulating the wire grid in PDMS permits the use of $\mathrm{Al}$ as the wire grid, as it prevents the $\mathrm{Al}$ from directly contacting the human body when integrated onto RGP contact lenses.

When light is incident on a WGP, transverse electric (TE) fields (light polarized along the direction of the $\mathrm{Al}$ lines) are reflected and transverse magnetic (TM) fields (light polarized perpendicular to the direction of the $\mathrm{Al}$ lines) are transmitted through the device. ${ }^{[4]}$ The extinction ratio (ER), defined as the ratio between TM and TE transmittance, is inversely proportional to the period of the NWGP. ${ }^{[5]}$ In this design, a $180 \mathrm{~nm}$ period is chosen for the $\mathrm{Al}$ lines as the wire grid must be subwavelength, less than $\lambda / 2(200 \mathrm{~nm}$ for visible light). Figure 2 shows simulation results for TM polarized transmission with Period, P, fixed at $180 \mathrm{~nm}$. These simulations are performed using rigorous coupled wave analysis (RCWA) with broad-band collimated light illuminating the sample with $\lambda=$ 400-800 nm supplied as the input. Figure 2(a) and (b) show contour plots of TM and TE transmission, respectively, through the NWGP for varying thicknesses of the $\mathrm{Al}$ and width, W, fixed at $100 \mathrm{~nm}$. It can be seen that high TM transmission is achieved with thicknesses between 90 and $140 \mathrm{~nm}$. For TE transmission the wire grid blocks greater than $99 \%$ of the light with Al thicknesses larger than $90 \mathrm{~nm}$. With these results an Al thickness of $100 \mathrm{~nm}$ was chosen. Fixing the height at $100 \mathrm{~nm}$ and then varying the width, Figure 2(c) and (d), shows strong performance for widths less than $110 \mathrm{~nm}$, for TM polarization. Additionally, widths greater than $80 \mathrm{~nm}$ sufficiently block TE transmission. Due to these results we chose to fabricate a device with $\mathrm{P}=$ $180 \mathrm{~nm}, \mathrm{H}=100 \mathrm{~nm}$, and $\mathrm{W}=100 \mathrm{~nm}$. A 1:1 aspect ratio in the Al structure will result in strong performance while avoiding fabrication complexities that arise with smaller line widths or higher aspect ratios.

Equally important for a WGP is its ability to maintain the transmission efficiency and polarization selectivity for varied angles of incidence. The angle tolerance of this design was analyzed to determine the field of view of the polarizer and is shown in Figure 2(e) and (f). To achieve angle-insensitivity, it is important that the incident TM wave is transmitted through the slits in the WGP rather than coupled to surface modes. ${ }^{[15]}$ The momentum matching equation for surface propagating modes through grating coupling is governed by the equation, $k_{\text {surface }}=k_{\text {incident }}+k_{\text {grating }}$, with $k_{\text {incident }}=k_{0} \sin (\theta)$ and $k_{\text {grating }}=\frac{2 \pi}{\Lambda} m$. Here, $\mathrm{k}_{0}$ is the incident field wave vector, $\mathrm{k}_{\text {surface }}$ is the wave vector of a field propagating along the surface of the grating ( $\mathrm{x}$-direction in Figure 1), $\theta$ is the incident angle with respect to normal incidence, $m$ is the diffraction order, and $\Lambda$ is the period of the grating. This surface propagating mode is sensitive to incident angle of light and should be avoided. ${ }^{[14]}$ As the period of the grating is reduced, the momentum provided from the grating is increased preventing momentum matching into the surface propagating mode ${ }^{[15]}$ and thereby allowing transmission through the $\mathrm{Al}$ wire grid. At $\Lambda=180 \mathrm{~nm}$, it is seen that the polarizer possesses strong performance for incident angles of $\pm 40^{\circ}$ which is comparable to advanced and complex HUD systems.

\section{Fabrication}

Methods for fabricating WGPs have been improving for many decades since Bird and Parrish first demonstrated an oblique angled evaporation to form infrared WGPs. ${ }^{[3]}$ NWGPs have been fabricated with a half-pitch as small as $50 \mathrm{~nm}$, extinction ratios in the tens of thousands, and transmission percentages greater than $90 \% \cdot{ }^{[16,17]}$ In this work, NIL is used to form an etch mask for a RIE into $\mathrm{Al}$ to form the metallic wire grids. A schematic of this process is outlined in Figure 3. Figure 4 shows a sample that followed this fabrication process without the intermediate a-Si layer to measure the performance of the device on glass before embedding the wire grid into PDMS. Figure 4(a) shows a large area SEM image with well-defined Al lines devoid of defects. Figure 4(b) shows a cross-sectional SEM image of the wire grid array and a close-up image of a few $\mathrm{Al}$ lines with dimensions of $P=180 \mathrm{~nm}, W=100 \mathrm{~nm}$, and $\mathrm{H}=100 \mathrm{~nm}$. The etch recipe results in a very straight side-wall profile. The 
TM Transmission

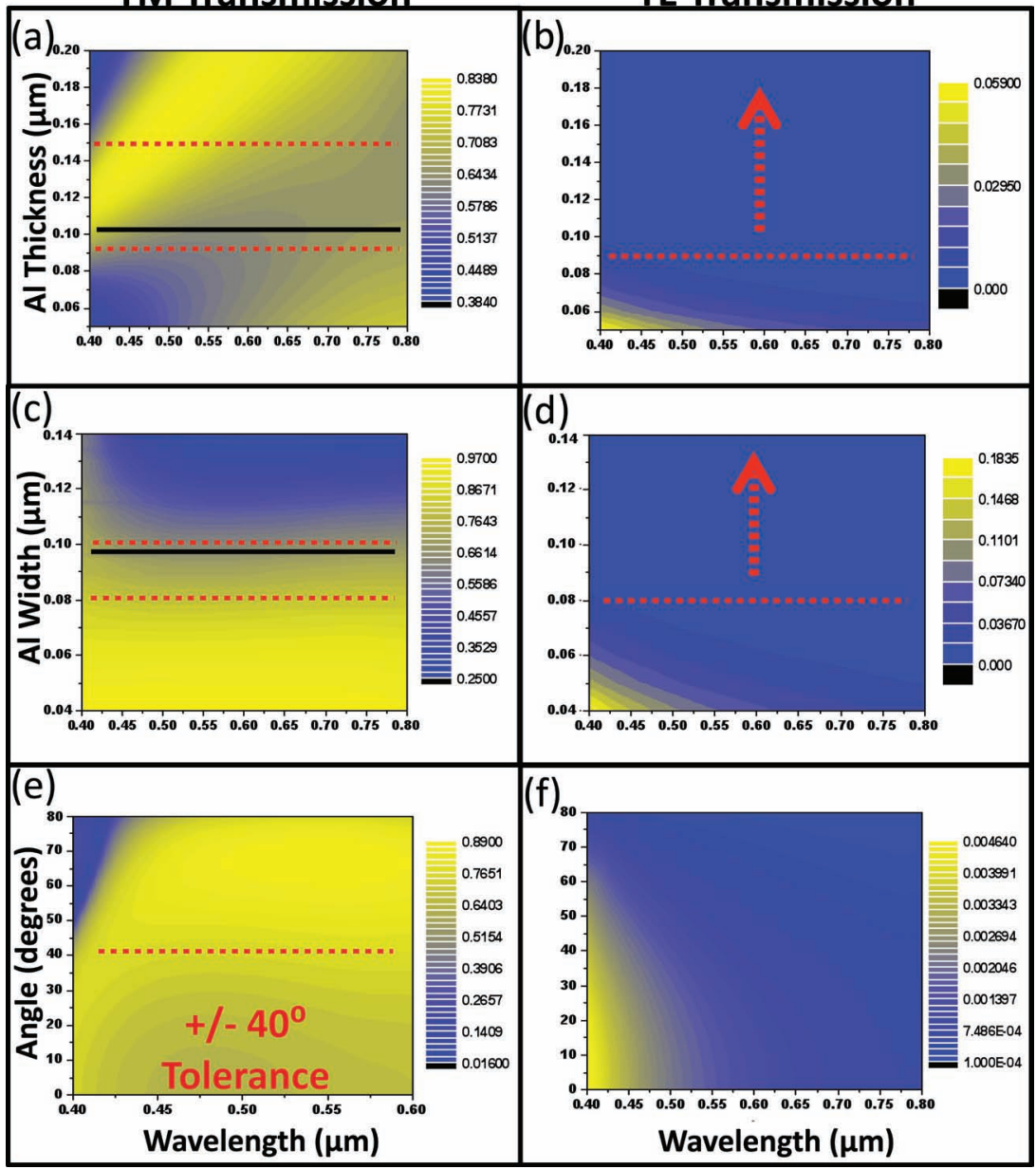

Figure 2. Transmission vs. wavelength through an AI WGP. Red dashed lines indicate acceptable performance range. Black solid line represents chosen device geometry. $\mathrm{P}=180 \mathrm{~nm}$. (a) TM and (b) TE polarized transmission with $\mathrm{W}=100 \mathrm{~nm}, \theta=0^{\circ}, \mathrm{H}$ is varied from $50-200 \mathrm{~nm}$. (c) TM and (d) TE polarized transmission with $\mathrm{H}=100 \mathrm{~nm}, \theta=0^{\circ}, \mathrm{W}$ is varied from $40-140 \mathrm{~nm}$. (e) TM and (f) TE polarized transmission with $\mathrm{H}=100 \mathrm{~nm}, \mathrm{~W}=100 \mathrm{~nm}, \theta$ is varied from $0^{\circ}$ to $80^{\circ}$.
Figure $4(\mathrm{c})$, it is seen that a minimum transmission of less than $1 \%$ was achieved for TE polarized light while a maximum transmission of greater than $60 \%$ was achieved for TM polarized light. The optical measurement was conducted at normal incidence using a Nikon TE300 inverted microscope with a halogen lamp for the light source. The numerical aperture of the collection optics is 0.04 to ensure high angular resolution. Figure 4(d) and (e) show the visual performance of the NWGP for TM and TE polarizations respectively. The display behind the device is a standard liquid crystal display (LCD), used to visually confirm the high contrast of transmitted light between TM and TE polarizations.

\section{Wire Grid Release}

Extensive experiments were performed to determine the most efficient way to release the Al wire grid from the carrier substrate. A gas-etch utilizing xenon di-fluoride $\left(\mathrm{XeF}_{2}\right)$ to remove a sacrificial a-Si layer was ultimately chosen as the most appropriate method due to the high selectivity of $\mathrm{XeF}_{2}$ between $\mathrm{Si}$ and $\mathrm{SiO}_{2} / \mathrm{Al}$. After the $\mathrm{Al} \mathrm{RIE}$ and substrate cleaning (steps 1-4 in Figure 3), PDMS is spin coated onto the sample to encapsulate the wire grid. It is important that the PDMS layer is sufficiently thin for subsequent integration onto contact lenses without greatly increasing the thickness of the lens. The $\mathrm{XeF}_{2}$ gas-etch is performed in a Xactix $\mathrm{XeF}_{2}$ etch tool, to undercut the a-Si and effectively release the encapsulated NWGP. The $\mathrm{XeF}_{2}$ gas is extremely selective between $\mathrm{Si}$ rounding on the top of the $\mathrm{Al}$ is a result of the poor selectivity (1:0.75) between the imprint resist and Al during the RIE. Analyzing the measured transmission spectrum of the Al WGP, and $\mathrm{SiO}_{2} / \mathrm{Al}$, with selectivity as high as 1000:1. ${ }^{[18]}$ We would like to emphasize that the a-Si layer is only $50 \mathrm{~nm}$ in thickness. Fluorine molecules are able to etch through this $50 \mathrm{~nm}$
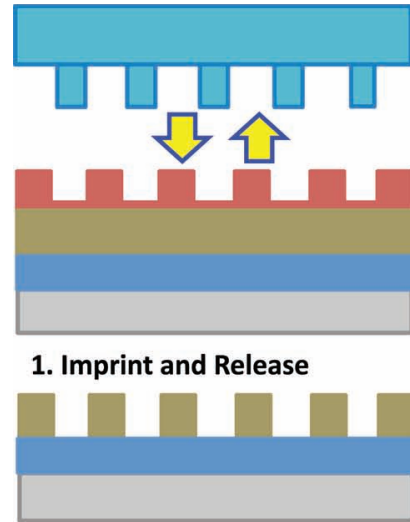

4. Resist Removal

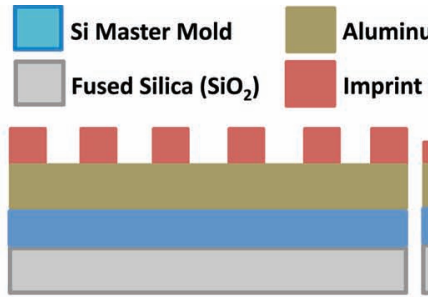

2. Residual Layer Etch

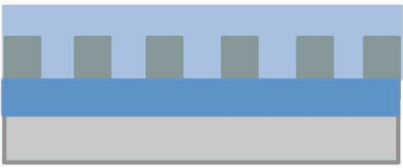

5. PDMS Encapsulation
a-Si PDMS

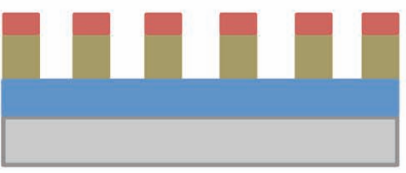

\section{Aluminum RIE}

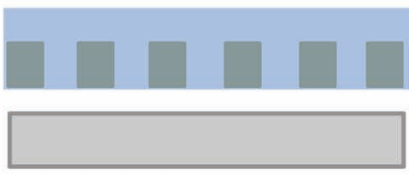

6. Selective a-Si Gas Etch

Figure 3. Fabrication steps to pattern an AI NWGP and further encapsulation in PDMSTy. 

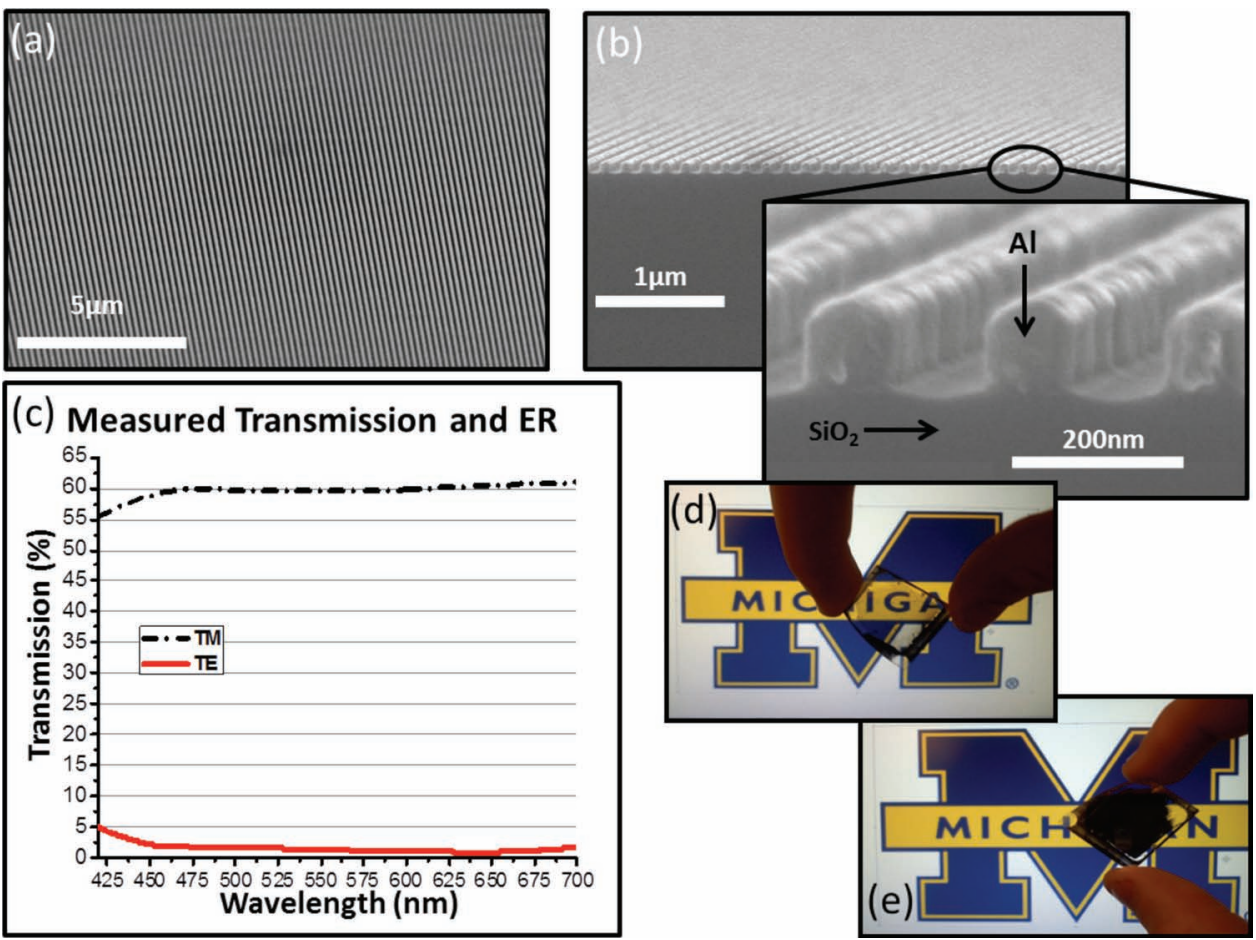

Figure 4. (a) Large area SEM of an Al NWGP fabricated on $\mathrm{SiO}_{2}, \mathrm{P}=180 \mathrm{~nm}, \mathrm{~W}=100 \mathrm{~nm}, \mathrm{H}=100 \mathrm{~nm}$. (b) Cross-sectional SEM and close up image of a few Al lines. (c) Measured TM (black dash-dot-dash line) and TE (solid red line) polarized transmission. (d) TM and (e) TE polarized visible image of the $180 \mathrm{~nm}$ period WGP performance in front of a LCD computer screen.

layer from the edge of the substrate and penetrate through the entire area of the substrate. This small thickness of a-Si leads to advantages in reducing material use. The $\mathrm{XeF}_{2}$ etch is performed at 5 miTorr with three etch cycles each with $600 \mathrm{~s}$ durations leading to a $30 \mathrm{~min}$ etch time to completely undercut the sample. This short etch time to release the structure is highly advantageous for manufacturing processes. After the $\mathrm{XeF}_{2}$ etch is complete, the PDMS film embedded with $\mathrm{Al}$ is placed upside down on a glass slide and the exposed Al side is coated with an additional $50 \mu \mathrm{m}$ of PDMS to completely encapsulate the NWGP in PDMS.

Figure 5(a) shows the measured transmission for TM and TE polarizations for a device successfully encapsulated in PDMS. This measurement was conducted in the same manner as in Figure 4(c). Analyzing the transmission spectrum it is seen that a minimum transmission of $6 \%$ was achieved for TE polarized
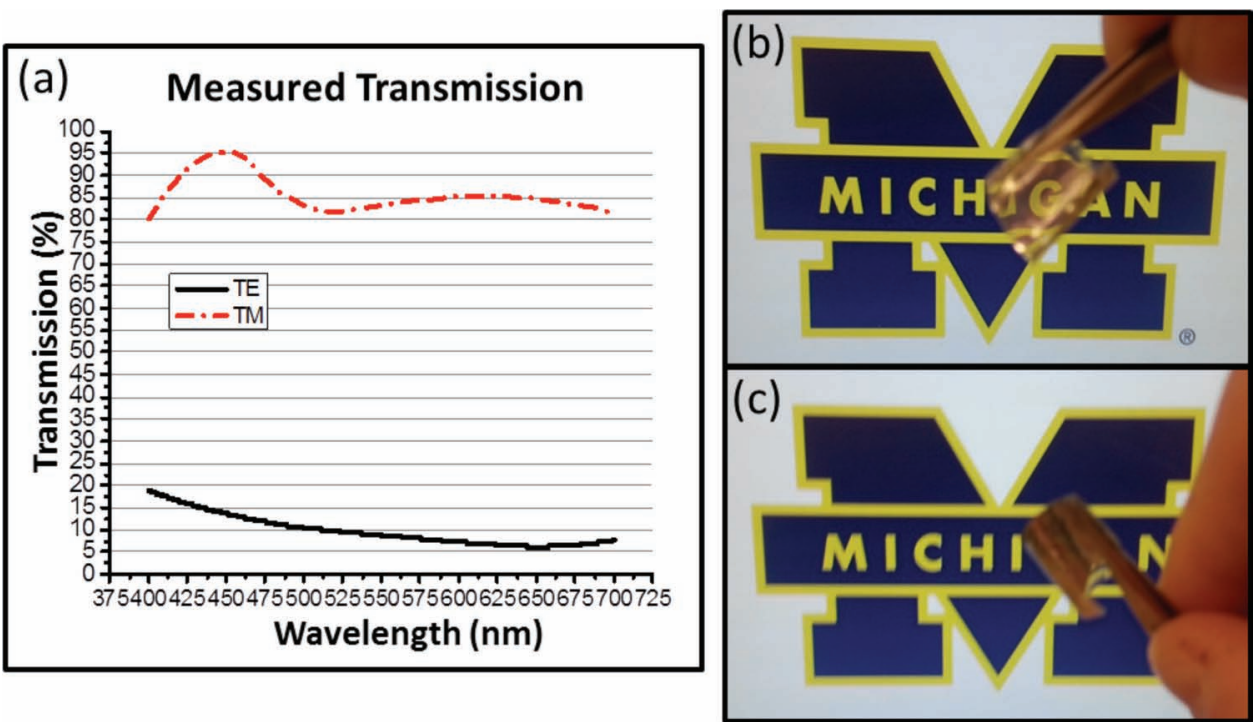

Figure 5. (a) Measured TM (red dash-dot-dash line) and TE (solid black line) polarized transmission of a Al NWGP embedded in PDMS. (c) TM and (c) TE polarized visible image of the WGP performance in front of a LCD computer screen. Note that the curvature of the sample indicates high flexibility. 
light while a maximum transmission of $95 \%$ was achieved for TM polarized light. The transmission for both TE and TM polarizations has increased in comparison to the device shown in Figure 4. Embedding a higher refactive index inside the metal slits effectively decreases the wavelength, making it less effective to cut off the TE propagation inside the metal slit waveguide, which is responsible for the increase in TE transmission. This increase is also partly attributed to loss of a small percentage of $\mathrm{Al}$ lines during the transfer into PDMS. This loss can be mitigated through incorporation of an adhesion promoter such as, GE SS4120, at the Al/PDMS interface as demonstrated by ${ }^{[19]}$ Figure $5(\mathrm{~b})$ and (c) show the visual performance of the flexible, gas permeable and biocompatible NWGP for TM and TE polarizations respectively. Again, the display behind the polarizer is a standard polarized LCD, used to visually confirm the high contrast of transmitted light between TM and TE polarizations. It is noticed in the images, by the curvature of the sample, that the embedded polarizer is extremely flexible and capable of accomodating a hemishperical surface while continuing to possess strong polarization performance.

\section{Oxygen Permeability}

Oxygen permeability (Dk), measured in $\mathrm{cm}^{2} \mathrm{~mL} \mathrm{O}_{2} / \mathrm{s} \mathrm{mL}$ $\mathrm{mmHg}$, and transmissibility (Dk/t), measured in $\mathrm{cm} \mathrm{mL} \mathrm{O}_{2} / \mathrm{s}$ $\mathrm{mL} \mathrm{mmHg}$, are important parameters describing the suitability of contact lenses. Insufficient delivery of oxygen to the human eye, less than $24 \times 10^{-9} \mathrm{Dk} / \mathrm{t}$, for extended periods of time can result in short and long term eye damage. ${ }^{[20,21]}$ In order to test the permeability of the NWGP embedded in PDMS, two samples were fabricated with the same $\mathrm{Al}$ dimensions as above $(\mathrm{P}=$ $180 \mathrm{~nm}, \mathrm{~W}=100 \mathrm{~nm}, \mathrm{H}=100 \mathrm{~nm}$ ) with two different thicknesses of PDMS, $200 \mu \mathrm{m}$ and $100 \mu \mathrm{m}$.

The samples were tested at Mocon Testing Laboratories (http://www.mocon.com) and measured on a MOCON Oxtran 2/21 Oxygen Permeability Instrument, which is certified according to the D-3985 standard test method for $\mathrm{O}_{2}$ transmission rates through polymer films. The conditions for this test are outlined in Table 1. The transmission rate of pure $\mathrm{O}_{2}$ is measured at $35^{\circ} \mathrm{C}$, as consistent with standard contact lens permeability measurements, with $\mathrm{N}_{2}$ and $\mathrm{H}_{2}$ as the carrier gas. As outlined in Table 2, the measured transmission rates of the two samples are 21500 and 42400 (cc/100 in ${ }^{2}$ day), respectively, for the $200 \mu \mathrm{m}$ and $100 \mu \mathrm{m}$ thick samples. Converting the transmission rates to permeability of $\mathrm{O}_{2}$ leads to values of $2.79 \cdot 10^{-9} \mathrm{Dk}$ and $5.51 \cdot 10^{-9} \mathrm{Dk}$ for the two devices. Transmissibility is defined as permeability normalized to the thickness of the device. Normalizing the two devices to their respective thicknesses leads to values of $5.58 \cdot 10^{-7} \mathrm{Dk} / \mathrm{t}$ and $5.51 \cdot 10^{-7} \mathrm{Dk} / \mathrm{t}$ showing consistent transmissibility between the two samples; and importantly, a transmissibility an order of magnitude higher than the minimum required to prevent eye damage.

\section{Conclusion}

A process for transferring wire grid polarizers into gas permeable biocompatible films has been developed. Reactive ion
Table 1. Testing conditions for measuring the transmission rate of oxygen.

\begin{tabular}{lc}
\hline Testing Conditions & \\
\hline Test Gas & Oxygen \\
Test Gas Concentration & $100 \% \mathrm{O}_{2}$ \\
Test Gas Humidity & $0 \% \mathrm{RH}$ \\
Test Gas Pressure & $760 \mathrm{mmHg}$ \\
Test Temperature & $35^{\circ} \mathrm{C}$ \\
Carrier Gas & $98 \% \mathrm{~N}_{2}, 2 \% \mathrm{H}_{2}$ \\
Carrier Gas Humidity & $0 \% \mathrm{RH}$ \\
\hline
\end{tabular}

Table 2. Measured $\mathrm{O}_{2}$ transmission rates and calculated $\mathrm{Dk}$ and $\mathrm{Dk} / \mathrm{t}$ for two samples with NWGPs embedded in $200 \mu \mathrm{m}$ and $100 \mu \mathrm{m}$ thick PDMS films.

\begin{tabular}{lcccc}
\hline Test Results & $\begin{array}{c}\mathrm{O}_{2} \text { Transmission } \\
\text { Rate } \mathrm{cc} / 100 \mathrm{in}^{2} \text { day }\end{array}$ & $\begin{array}{c}\text { Thickness } \\
{[\mu \mathrm{m}]}\end{array}$ & $\begin{array}{c}\mathrm{Dk} \mathrm{cm} \mathrm{mL}^{2} \\
\mathrm{O}_{2} / \mathrm{s} \mathrm{mL} \mathrm{mmHg}\end{array}$ & $\begin{array}{c}\mathrm{Dk} / \mathrm{t} \mathrm{cm} \mathrm{mL} \\
\mathrm{O}_{2} / \mathrm{s} \mathrm{mL} \mathrm{mmHg}\end{array}$ \\
\hline WGP-GP-1 & 21500 & 200 & $2.79 \cdot 10^{-9}$ & $5.58 \cdot 10^{-7}$ \\
WGP-GP-2 & 42400 & 100 & $5.51 \cdot 10^{-9}$ & $5.51 \cdot 10^{-7}$ \\
\hline
\end{tabular}

etching of aluminium through a nanoimprint defined polymer mask is used as a fabrication method for defining the polarizer. Extremely selective gas etching allows $50 \mathrm{~nm}$ thin films of a-Si to be laterally etched, releasing the wire grid for increased delamination yield. Full encapsulation of these wire grid polarizers into PDMS offers a solution for integration onto RGP contact lenses. The optical performance can be improved by further reducing the period of the Al grating, which in this work is limited to $180 \mathrm{~nm}$ period mold available in our lab. It should be noted that these methods can be extended towards complex optical and electronic devices for integration into flexible, gas permeable, and/or biocompatible systems for advanced display technologies and integrated medical instruments while maintaining fabrication processes on rigid substrates.

\section{Experimental Section}

In fabrication, a $50 \mathrm{~nm}$ thick amorphous silicon (a-Si) sacrificial layer is first deposited by Plasma enhanced chemical vapor deposition (PECVD) on the $\mathrm{SiO}_{2}$ substrate followed by an e-beam evaporation of $100 \mathrm{~nm}$ of Al. To fabricate an etch mask for the Al RIE, NIL was performed into MRI-8030, a thermally curable resist. The resist is diluted with a PGMEA based solvent in order to achieve an optimal spin coat thickness for imprinting and generate a minimal residual layer at the bottom of the imprinted areas. It is important to reduce the thickness of the imprinted residual layer as much as possible in order to minimize the etch time necessary to remove it and effectively control the line width of the etched Al. Prior to imprinting, the master mold is cleaned and then vapor coated with $1 \mathrm{H}, 1 \mathrm{H}, 2 \mathrm{H}, 2 \mathrm{H}$-perfluorodecyltrichlorosilane (FDTS) at a temperature of $140{ }^{\circ} \mathrm{C}$ for a duration of $40 \mathrm{~min}$ in a FDTS saturated environment to uniformly deposit the fluoro-polymer. This FDTS acts as an anti-adhesion, release layer between the master mold and imprinting resist. ${ }^{[22]}$ The nanoimprint is performed using a Nanonex 2000 imprinting tool for $2 \mathrm{~min}$ at $500 \mathrm{psi}$ and $120^{\circ} \mathrm{C}$. After de-molding the master mold from the resist, the inherent residual layer from the 
imprint process is removed with an $\mathrm{O}_{2}$ RIE $\left(20 \mathrm{sccm} \mathrm{\textrm {O } _ { 2 }}, 30 \mathrm{~W}\right.$ RF Bias, $100 \mathrm{~W}$ TCP Bias, $12 \mathrm{mTorr}$ ). The Al etch is performed directly after the residual layer is removed. First, $\mathrm{BCl}_{3}$ is used to remove the $\mathrm{Al}$ oxide from the thin film ( $40 \mathrm{sccm} \mathrm{BCl}$, $100 \mathrm{~W}$ RF Bias, $200 \mathrm{~W}$ TCP Bias, $10 \mathrm{mTorr}$ ) and then $\mathrm{Cl}_{2}$ is used to etch the $\mathrm{Al}(12 \mathrm{sccm} \mathrm{Cl}, 20 \mathrm{sccm} \mathrm{BCl}, 20 \mathrm{sccm}$ Ar, $50 \mathrm{sccm} \mathrm{He}, 50$ W RF Bias, 200 W TCP Bias, $10 \mathrm{mTorr})$. Finally the sample is submersed in acetone, with ultrasonic agitation to remove the remaining resist from the top of the Al grating, completing the fabrication of the Al wire grid (steps 1-4 in Figure 4).

Dow Corning Sylgard 184 base and curing agent is mixed at a ratio of 10:1 to synthesize the PDMS compound. PDMS is deposited on the sample and spun at $1000 \mathrm{rpm}$ to generate a film thickness of $50 \mu \mathrm{m}$. The coated sample is subsequently baked at $120^{\circ} \mathrm{C}$ on a hot plate for $40 \mathrm{~min}$ to cure the PDMS.

\section{Acknowledgements}

This work is supported by the DARPA SCENICC program through a subcontract from Innovega Inc.

Received: January 25, 2013

Published online: March 18, 2013

[1] H. Ho, E. Saeedi, S. S. Kim, T. T. Shen, B. A. Parviz, Mems 2008: 21 st IEEE Int. Conf. on Microelectromechanical Systems, Technical Digest, 2008, pp.403-406.

[2] J. Pandey, T. T. Liao, A. Lingley, R. Mirjalili, B. A. Parviz, B. P. Otis, IEEE Trans. Biomed. Circuits Systems 2010, 4, 454-461.

[3] G. R. Bird, M. Parrish, J. Optical Soc. Am. 1960, 50, 886-891.
[4] E. Hecht, Optics, 4th Ed., Addison-Wesley, Texas 2002, pp.325-378.

[5] S. W. Ahn, K. D. Lee, J. S. Kim, S. Hw. Kim, J. D. Park, S. H. Lee, Nanotechnology 2005, 16, 1874-1877.

[6] L. Chen, J. J. Wang, F. Walters, X. Deng, M. Buonanno, S. Tai, X. M. Liu, J. Vacuum Sci. Technol. B 2007, 25, 2654-2657.

[7] L. Chen, J. J. Wang, F. Walters, X. Deng, M. Buonanno, S. Tai, X. M. Liu, Appl. Phys. Lett. 2007, 90, 1-3.

[8] Y. J. Shin, C. Pina Hernandez, Y. K. Wu, J. G. Ok, L. J. Guo, Nanotechnology 2012, 23, 1-6.

[9] Z. N. Yu, W. Wu, L. Chen, S. Y. Chou, J. Vacuum Sci. Technol. B 2001, 19, 2816-2819.

[10] T. I. Kim, S. M. Seo, Nanotechnology 2009, 20, 1-6.

[11] M. A. Meitl, X. T. Zhu, V. Kumar, K. J. Lee, X. Feng, Y. Y. Huang, I. Adesida, R. G. Nuzzo, J. A. Roger, Nat. Mater. 2006, 5, 33-38.

[12] S. W. Ahn, K. D. Lee, J. S. Kim, S. H. Kim, S. H. Lee, J. D. Park, P. W. Yoon, Microelectron. Engin. 2005, 78-79, 314-318.

[13] C. Chen, P. L. Niu, C. K. Sung, C. H. Chen, Microelectron. Engin. 2013, 102, 53-59.

[14] Y.-K. Wu, A. E. Hollowell, C. Zhang, L. J. Guo, Sci. Rep. 2013, 3, 1-6.

[15] J. Zhou, L. J. Guo, unpublished.

[16] Y. Ikinci, H. H. Solak, C. David, H. Sigg, Opt. Express 2006, 14, 2323-2334.

[17] J. J. Wang, F. Walters, X. M. Liu, P. Sciortino, X. G. Deng, Appl. Phys. Lett. 2007, 90, 1-3.

[18] H. F. Winters, J. W. Coburn, Appl. Phys. Lett. 1979, 34, 70-73.

[19] L. Kersey, V. Ebacher, V. Bazargan, R. Z. Wang, B. Stoeber, Lab Chip 2009, 9, 1002-1004.

[20] J. Hadassah, P. K. Sehgal, Clin. Exp. Optom. 2006, 89, 374-380.

[21] B. A. Holden, G. W. Mertz, Investigative Ophthalmol. Visual Sci. 1984, 25, 1161-1167.

[22] L. J. Guo, Adv. Mater. 2007, 19, 495-513. 